\title{
NONHOMOGENEOUS RANDOM WALKS SYSTEMS ON $\mathbb{Z}$
}

\author{
ELCIO LEBENSZTAYN ${ }^{* * *}$ AND \\ FÁBIO PRATES MACHADO, ${ }^{* * * *}$ University of São Paulo \\ MAURICIO ZULUAGA MARTINEZ, ${ }^{* * * *}$ Federal University of Pernambuco
}

\begin{abstract}
We consider a random walks system on $\mathbb{Z}$ in which each active particle performs a nearestneighbor random walk and activates all inactive particles it encounters. The movement of an active particle stops when it reaches a certain number of jumps without activating any particle. We prove that if the process relies on efficient particles (i.e. those particles with a small probability of jumping to the left) being placed strategically on $\mathbb{Z}$, then it might survive, having active particles at any time with positive probability. On the other hand, we may construct a process that dies out eventually almost surely, even if it relies on efficient particles. That is, we discuss what happens if particles are initially placed very far away from each other or if their probability of jumping to the right tends to 1 but not fast enough.
\end{abstract}

Keywords: Random walk; epidemic model; frog model

2010 Mathematics Subject Classification: Primary 60K35

Secondary 60G50

\section{Introduction}

We study a random walks system on $\mathbb{Z}$ whose parameters are the integers $L \geq 1$ and $N \geq 1$, and a sequence $\left\{q_{n}\right\}_{n \geq 1}$ of real numbers in $(0,1)$. At time 0 , there are $N$ particles at each vertex of $\mathbb{N}=\{1,2, \ldots\}$. All particles are inactive, except for those placed at the vertex 1 . The active particles move as discrete-time independent nearest-neighbor random walks on $\mathbb{Z}$, and activate the inactive particles encountered during their trajectories. Each active particle has a lifetime which depends on the past of the process, in the sense that its displacement lasts until it reaches a total of $L$ jumps without activating any particle. When several active particles simultaneously jump on an inactive particle, we consider all of them responsible for the activation. We suppose that the jump probabilities of the active particles depend on their initial position: particles initially placed at position $n$, in the event of activation, jump at each step to the right with probability $1-q_{n}$ or to the left with probability $q_{n}$.

This model, named the particle process on $\mathbb{Z}$, was first introduced in [5]. Its dynamics describe the evolution of a virus in a population with an infinite number of individuals (say, computers) connected in line. Under this interpretation, the active particles are viruses which create $N$ replicas every time a new computer is infected. Once this happens, this computer

Received 27 April 2009; revision received 11 March 2010.

* Postal address: Department of Statistics, Institute of Mathematics and Statistics, University of São Paulo, Rua do Matão 1010, CEP 05508-090, São Paulo, Brazil.

** Email address: elcio@ime.usp.br

*** Email address: fmachado@ime.usp.br

**** Postal address: Department of Statistics, Federal University of Pernambuco, Cidade Universitária, CEP 50740-

540, Recife, PE, Brazil. Email address: zuluaga@ime.usp.br 
activates an antivirus. The parameter $L$ is the initial number of lives (the resistance) a virus has, in the sense that it loses one life (weakens one unit) whenever it jumps to a computer with an antivirus. The main purpose is to study whether the process survives (globally), that is, whether there is a positive probability that an infinite number of individuals become infected. In [5], the survival issue was investigated for this and similar models. Kurtz et al. [4] studied this model on the complete graph and stated limit theorems for the proportion of visited vertices. The question of local survival (infinite visits of active particles to the origin) in a model on $\mathbb{Z}$ was studied by Gantert and Schmidt [3]. For problems related to global survival of branching random walks on graphs, we refer the reader to Bertacchi and Zucca [1]. They also addressed questions related to local survival.

The paper is organized as follows. Section 2 carries the main results, namely a sufficient condition for the process to survive with positive probability (Theorem 2.1) and a sufficient condition for the process to die out (Theorem 2.2). Furthermore, in Section 2 we present important remarks and examples which make clear why the hypotheses of these two theorems are relevant for the process. Section 3 brings the proofs for the main results together with auxiliary lemmas, mostly presenting useful inequalities. Section 4 is devoted to a generalization and to a nonstandard application of our results.

\section{Main results}

Among the main questions related to this type of process are those based on the dichotomy between survival and disappearance of the agents (active particles). Intuitively speaking, to envision the survival event, what we need is an infinite chain of particles, not too far away from each other, whose probability of jumping to the right grows steadily. Pushing the cloud of active particles to the right, they would guarantee the survival of the process (with positive probability) even though all the other particles do a poor job. This is what we present in the next theorem.

Let $b_{0}=0$, and let $\left\{b_{i}\right\}_{i \in \mathbb{N}}$ be a positive bounded sequence of integer numbers. The idea is to split the set $\mathbb{N}$ into disjoint blocks of sizes $b_{1}, b_{2}, \ldots$ and to ensure that there is an efficient particle at each block. Note that $l_{n}$, the leftmost vertex, and $r_{n}$, the rightmost vertex, in block $n$ are given by

$$
l_{n}=\sum_{j=0}^{n-1} b_{j}+1 \quad \text { and } \quad r_{n}=\sum_{j=0}^{n} b_{j} .
$$

Theorem 2.1. Suppose that $l_{n}$ and $r_{n}$ are as in (2.1), and that

$$
\bar{q}_{n}=\min \left\{q_{l_{n}}, q_{l_{n}+1}, \ldots, q_{r_{n}}\right\} .
$$

If $\sum_{n=0}^{\infty}\left(\bar{q}_{n}\right)^{M}<\infty$ for some $M>0$ then the particle process on $\mathbb{Z}$ survives with positive probability, no matter what the values of $N$ and $L$.

Observe that if $b_{i}=1$ for all $i$ then the condition for survival is $\sum_{n=1}^{\infty}\left(q_{n}\right)^{M}<\infty$ for some $M>0$.

The following result (see, e.g. [2, p. 422]) is useful for what comes next.

Lemma 2.1. Let $\left\{a_{n}\right\}_{n \geq 1}$ be a sequence of real numbers in $(0,1)$. Then,

$$
\prod_{n=1}^{\infty}\left(1-a_{n}\right)=0 \Longleftrightarrow \sum_{n=1}^{\infty} a_{n}=\infty .
$$


Now we present an example such that, by a simple calculation, it is possible to conclude that the particle process survives.

Example 2.1. Let $k \in \mathbb{N}$, and consider the particle process with

$$
q_{n}= \begin{cases}\frac{1}{(a+2)^{2}} & \text { if } n=1+a k, a=0,1,2, \ldots \\ 1 & \text { otherwise. }\end{cases}
$$

We can present an explicit lower bound for the survival probability. Let $\{i \rightarrow j\}$ be the event that an active particle originally placed at vertex $i$ visits vertex $j$. Then,

$$
\begin{aligned}
\mathrm{P}(\text { survival }) & \geq \mathrm{P}(1 \rightarrow k+1) \mathrm{P}(k+1 \rightarrow 2 k+1) \mathrm{P}(2 k+1 \rightarrow 3 k+1) \cdots \\
& \geq\left(1-\frac{1}{2^{2}}\right)^{k}\left(1-\frac{1}{3^{2}}\right)^{k}\left(1-\frac{1}{4^{2}}\right)^{k} \ldots \\
& >0
\end{aligned}
$$

The last inequality follows from Lemma 2.1. Besides, observe that the hypothesis of Theorem 2.1 holds for this case. In some sense, this very simple computation is the basic idea behind the proof of that result.

Remark 2.1. Theorem 2.1 asserts that the propagation event may occur if the process counts on very efficient individuals placed at appropriate positions, even if all other individuals jump to the left with probability 1 . Observe that the result holds even for the worst case, namely the case $L=N=1$. If we consider the following small modifications in the model:

(i) allow sites with no particles at time 0 ; and

(ii) introduce the feature that one life is lost only when live particles jump to sites which have been visited before (instead of not activating any particle),

then Theorem 2.1 still holds.

Remark 2.2. Besides modification (ii), consider

(iii) breaking the set of vertices into blocks of size $K$ (any positive integer), placing just one particle per block (anywhere in each block) with $L=1$.

Denote the probability of jumping to the left for a particle originally placed in block $i$ by $q_{i}$. As a consequence of Theorem 2.1, no matter where each particle is placed inside each block and the value of $K$, if $\sum_{i=1}^{\infty} q_{i}{ }^{M}<\infty$ for some $M>0$ then the survival probability is positive.

Remark 2.3. Even with modification (ii), there is no guarantee of survival if the sizes of the blocks in modification (iii) grow. To this aim, consider $\mathbb{N}$ partitioned by a set of growing adjacent blocks, the size of block $i=1,2,3, \ldots$ being $i^{i}$. At time 0 place one particle per block at position $R_{i}$, such that $R_{1}=1, R_{2}=2$, and $R_{i}=R_{i-1}+(i-1)^{i-1}$ for $i \geq 3$. Now consider $L=1$ and a particle process whose probabilities of jumping to the left are defined by

$$
q_{R_{i}}=\frac{1}{(i+1)^{2}} .
$$


Let us define $A_{R_{i}}$ to be the particle originally placed at vertex $R_{i}$ that is activated. The process $\left\{q_{R_{i}}\right\}$ dies out almost surely (a.s.) since

$$
\mathrm{P}\left(A_{R_{i}}\right) \leq \sum_{j=1}^{i-1} \mathrm{P}\left(R_{j} \rightarrow R_{i}\right)=\sum_{j=1}^{i-1}\left(1-\frac{1}{(j+1)^{2}}\right)^{R_{i}-R_{j}}<\frac{i}{\mathrm{e}^{i}} .
$$

Observe now that, for a set of positions $\left\{R_{i}\right\}$, we can generate probabilities of jumping to the left-say $\left\{\bar{q}_{R_{i}}\right\}$-for a set of particles placed at these positions, such that the associated process survives with positive probability. This is because

$$
\mathrm{P}(\text { survival }) \geq \prod_{i=1}^{\infty} \mathrm{P}\left(R_{i} \rightarrow R_{i+1}\right)=\prod_{i=1}^{\infty}\left(1-\bar{q}_{R_{i}}\right)^{R_{i+1}-R_{i}} .
$$

To finish, take $a_{i} \in(0,1)$ such that $\sum_{i=1}^{\infty} a_{i}<\infty$ and $\bar{q}_{R_{i}}=a_{i} /\left(R_{i+1}-R_{i}\right)$.

As a consequence of Lemma 2.1,

$$
\prod\left(1-a_{n}\right)^{j_{n}}>0 \Longleftrightarrow \sum j_{n} a_{n}<\infty,
$$

and, from this, the right-hand side of (2.3) is positive. This shows that the process survives with positive probability. Finally, observe that $\sum_{i=1}^{\infty}\left(q_{R_{i}}\right)^{M}<\infty$ and $\sum_{i=1}^{\infty}\left(\bar{q}_{R_{i}}\right)^{M}<\infty$ for all $M \geq 1$.

The next result, however, states that no matter how large $N$ and $L$ are the condition $\lim _{n \rightarrow \infty} q_{n}=0$ is not sufficient for the survival of the particle process. In fact, we prove that if $q_{n}$ decreases to 0 , but not sufficiently fast, then the process dies out a.s.

Theorem 2.2. The particle process on $\mathbb{Z}$ dies out a.s. if, for some $\alpha>0$ and $I>0$,

$$
\frac{1}{i^{\alpha}} \leq \min \left\{q_{1}^{N L}, q_{2}^{N L}, \ldots, q_{i^{i}}^{N L}\right\}
$$

for all $i \geq I$.

Remark 2.4. For any $M>0$ fixed, the particle process on $\mathbb{Z}$ dies out a.s. if, for large $i$,

$$
q_{i}=\frac{1}{(\ln i)^{M}} .
$$

We conclude from Theorem 2.2 that the particle process on $\mathbb{Z}$ dies out a.s. if $q_{n}=q \in(0,1)$ for all $n \geq 1$ or if $\inf _{n} q_{n}>0$.

Next we present two examples showing that the survival of the process is a matter of having efficient particles placed at strategic vertices. The first example shows in particular that it is not enough to have $\lim _{n \rightarrow \infty} q_{n}=0$ for the process to survive.

Example 2.2. Let $q>0$, and consider the particle process with

$$
q_{i}= \begin{cases}q & \text { if } i<100 \\ \frac{1}{\ln i} & \text { otherwise. }\end{cases}
$$

Observing that $\left(\ln \left(i^{i}\right)\right)^{N L}<i^{N L+1}$ for large enough $i$ and applying Theorem 2.2, we conclude that this particle process dies out a.s. 
Now, from Remark 2.2, it is possible to make a small modification in the set up of Example 2.2 and obtain the survival of the model. A simple way to do this is to split the set of vertices into blocks of size $K$, placing at the end of each block (vertices $i K$ for all $i$ ) a particle which jumps to the left with probability $i^{-1}$. These are the efficient particles which make the survival of the process possible, even in the worst case.

Another small modification and the process is ruined, not being able to survive. The efficient particles are still there, but they are placed far away from each other, turning the whole process into a process which dies out, no matter how large $N$ and $L$ are.

Example 2.3. Let $N$ and $L$ be positive integers, let $q>0$, and consider the particle process with

$$
q_{n}= \begin{cases}q & \text { if } n<100 \\ \frac{1}{i} & \text { if } n=i^{i}, i=1,2, \ldots, \\ \frac{1}{\ln n} & \text { otherwise. }\end{cases}
$$

From Theorem 2.2 we conclude that this process dies out a.s.

\section{Proofs}

We start this section with a technical but unusual inequality. It is useful in the proof of Theorem 2.1.

Lemma 3.1. For $i \in\{1, \ldots, M\}$, let $a_{i-1}=\prod_{m=1}^{i-1}\left(1-d_{m}\right)$ and $a_{0}=1$. If $1-c_{i} \leq d_{i}<1$ for all $i$ then

$$
\sum_{i=1}^{M} d_{i} a_{i-1} \geq 1-\prod_{i=1}^{M} c_{i}
$$

Proof. Let $\bar{c}_{i}=1-d_{i}$ (note that $1 \geq c_{i} \geq \bar{c}_{i}$ ). Then

$$
\sum_{i=1}^{M} d_{i} \prod_{m=1}^{i-1}\left(1-d_{m}\right)=\sum_{i=1}^{M}\left(1-\bar{c}_{i}\right) \prod_{m=1}^{i-1} \bar{c}_{m}=1-\prod_{i=1}^{M} \bar{c}_{i} \geq 1-\prod_{i=1}^{M} c_{i} .
$$

Next we prove Theorem 2.1. The idea of the proof is to construct an event that guarantees the survival of the particle process, showing that this event has positive probability. Think of a chain reaction, such that the survival of the particle process depends only on the leftmost particle placed in each block $i$, whose jump probability to the left is $\bar{q}_{i}$ for every $i$. If these particles keep being activated and activating without stopping, then the particle process survives.

For future use, observe that, under the conditions of Theorem 2.1,

$$
\bar{q}_{n-j} \rightarrow 0 \quad \text { as } n \rightarrow \infty \text { for all } j .
$$

Next we define, for $j<i$ and $n \in \mathbb{N}$, the following events.

$$
\begin{aligned}
& \bar{A}_{i} \quad \text { The leftmost particle in block } i \text {, with a jump probability to the left of } \bar{q}_{i} \text {, } \\
& \left\{B_{j} \Rightarrow B_{i}\right\} \quad \text { The leftmost particle with a jump probability to the left of } \bar{q}_{j} \text { in block } j \\
& \text { activates the leftmost particle with a jump probability to the left of } \bar{q}_{i} \\
& \text { in block } i \text {. } \\
& \left\{B_{j} \nRightarrow B_{i}\right\} \quad\left\{B_{j} \Rightarrow B_{i}\right\}^{\mathrm{c}} \text {. }
\end{aligned}
$$


Furthermore, let $b=\max \left\{b_{1}, b_{2}, \ldots\right\}$ and $d_{j}=\mathrm{P}\left(B_{n-j} \Rightarrow B_{n}\right)$. From (2.2), observe that

$$
d_{j} \geq\left(1-\bar{q}_{n-j}\right)^{b_{n-1}+\cdots+b_{n-j}} \geq\left(1-\bar{q}_{n-j}\right)^{j b} \geq 1-c_{j},
$$

where the last step is Bernoulli's inequality for $c_{j}=j b \bar{q}_{n-j}$. Therefore, by (3.1), $d_{j} \geq$ $1-c_{j}>0$ for large enough $n$.

Proof of Theorem 2.1. We prove that, for some $\delta>0$ and any integer $M$ such that the series in the hypothesis converges,

$$
\mathrm{P}\left(A_{n+M}\right) \geq \delta \mathrm{P}\left(A_{M}\right) \text { for all } n .
$$

As $\mathrm{P}\left(A_{M}\right)>0$ for all $M$, just let $n$ go to $\infty$ in (3.2) to complete the proof. It is enough to prove (3.2) in the case $N=L=1$.

First observe that $\bar{A}_{n}$ occurs if

$$
\left\{\bar{A}_{n-j}, B_{n-j} \Rightarrow B_{n}, B_{n-j+1} \nRightarrow B_{n}, B_{n-j+2} \nRightarrow B_{n}, \ldots, B_{n-1} \nRightarrow B_{n}\right\}
$$

occurs for some $j<n$. From this, with $\dot{\cup}$ meaning the disjoint union, we have

$$
\begin{aligned}
\bar{A}_{n} \supset\left\{\bar{A}_{n-1}, B_{n-1} \Rightarrow B_{n}\right\} & \dot{\cup}\left\{\bar{A}_{n-2}, B_{n-2} \Rightarrow B_{n}, B_{n-1} \nRightarrow B_{n}\right\} \\
& \dot{\cup}\left\{\bar{A}_{n-3}, B_{n-3} \Rightarrow B_{n}, B_{n-2} \nRightarrow B_{n}, B_{n-1} \nRightarrow B_{n}\right\} \dot{\cup} \ldots \\
& \dot{\cup}\left\{\bar{A}_{n-M}, B_{n-M} \Rightarrow B_{n}, B_{n-M+1} \nRightarrow B_{n}, \ldots, B_{n-1} \nRightarrow B_{n}\right\} .
\end{aligned}
$$

This implies that, for $C=b^{M} M !$

$$
\begin{aligned}
\mathrm{P}\left(\bar{A}_{n}\right) & \geq \mathrm{P}\left(\bar{A}_{n-1}\right)\left(d_{1}+d_{2}\left(1-d_{1}\right)+d_{3}\left(1-d_{2}\right)\left(1-d_{1}\right)+\cdots\right. \\
& \left.\quad+d_{M}\left(1-d_{M-1}\right) \cdots\left(1-d_{1}\right)\right) \\
& \geq \mathrm{P}\left(\bar{A}_{n-1}\right)\left(1-C \bar{q}_{n-1} \bar{q}_{n-2} \cdots \bar{q}_{n-M}\right) \\
& >0 .
\end{aligned}
$$

While the second inequality in the above display is justified by Lemma 3.1, the last inequality holds by the definition of $\bar{A}_{n}$ and by (3.1) for large enough $n$. Now, iterating the above display $n$ times, starting from $\mathrm{P}\left(\bar{A}_{n+M}\right)$, observing that $\sum_{j=M+1}^{\infty} \bar{q}_{j-1} \bar{q}_{j-2} \cdots \bar{q}_{j-M}<\infty$ if $\sum_{n=0}^{\infty}\left(\bar{q}_{n}\right)^{M}<\infty$ and using Lemma 2.1, we reach (3.2). This completes the proof of Theorem 2.1.

In order to prove Theorem 2.2 , let $\mathcal{R}_{i}, i \in \mathbb{N}$, be the set of all vertices visited by particles placed originally at vertex $i$, during their virtual lives. We use the word 'virtual' because we do not know whether vertex $i$ is visited by some particle; the set $\mathcal{R}_{i}$ becomes 'real' if $i$ is actually visited. For $i, j \in \mathbb{N}$, we define the following events: $A_{i}:=$ the particles at vertex $i$ are activated and $\{i \nrightarrow j\}:=\{i \rightarrow j\}^{\mathrm{c}}$.

\subsection{Proof of Theorem 2.2}

We consider the following modification in the particle process: we assume that an active particle loses one life whenever it jumps toward the origin. In this new process, each active particle has a life which depends only on its own random walk, so that we call it the independent particle process. Clearly, Theorem 2.2 is proved once we show that, under hypothesis (2.4), the independent particle process dies out a.s. 
First we consider the case in which $\alpha=1$ (which implies that Theorem 2.2 holds for every $\alpha \leq 1)$. Let

$$
M_{i}:=i^{3 i / 8+1} \text { and } S_{i}:=i^{2} M_{i},
$$

and observe that, for large $i$, we have $i^{i}>M_{i} S_{i}$.

Lemma 3.2. Under hypothesis (2.4), we have, for the independent particle process and large enough $i$,

$$
\mathrm{P}\left(A_{i^{i}}\right) \leq i\left(1-i^{-1}\right)^{S_{i}} M_{i}+\left(1-\left(i^{-1}\right)^{3 i / 8}\right)^{M_{i}} .
$$

Observe that (3.3) suffices to prove that $\lim _{n \rightarrow \infty} \mathrm{P}\left(A_{n}\right)=0$ (since $A_{n}$ is a decreasing sequence of events), meaning that the independent particle process dies out a.s.

In order to prove Lemma 3.2, for $J_{i} \in\left\{i^{i}, i^{i}-S_{i}, \ldots, i^{i}-M_{i} S_{i}\right\}$, we define the events

$$
B_{J_{i}}=\bigcup_{j=1}^{J_{i}-S_{i}-1}\left\{j \rightarrow J_{i}\right\} \quad \text { and } \quad C_{J_{i}}=\bigcup_{j=J_{i}-S_{i}}^{J_{i}-1}\left\{j \rightarrow J_{i}\right\} .
$$

In the sequel, we will show the next result.

Lemma 3.3. For large enough $i$,

$$
\begin{aligned}
& \mathrm{P}\left(B_{J_{i}}\right) \leq i\left(1-i^{-1}\right)^{S_{i}}, \\
& \mathrm{P}\left(C_{J_{i}}\right) \leq 1-\left(i^{-1}\right)^{3 i / 8} .
\end{aligned}
$$

Let us first see how this result implies Lemma 3.2.

Proof of Lemma 3.2. Note that

$$
A_{i^{i}} \subset A_{i^{i}-S_{i}} \cap\left\{B_{i^{i}} \cup C_{i^{i}}\right\} \subset B_{i^{i}} \cup\left\{A_{i^{i}-S_{i}} \cap C_{i^{i}}\right\} ;
$$

thus, as the particles act independently in the independent particle process,

$$
\mathrm{P}\left(A_{i^{i}}\right) \leq \mathrm{P}\left(B_{i^{i}}\right)+\mathrm{P}\left(A_{i^{i}-S_{i}}\right) \mathrm{P}\left(C_{i^{i}}\right) .
$$

Hence, using (3.4) and (3.5), we obtain, for large enough $i$,

$$
\mathrm{P}\left(A_{i^{i}}\right) \leq i\left(1-i^{-1}\right)^{S_{i}}+\mathrm{P}\left(A_{i^{i}-S_{i}}\right)\left(1-\left(i^{-1}\right)^{3 i / 8}\right) .
$$

Observe that

$$
\begin{aligned}
\mathrm{P}\left(A_{i^{i}-S_{i}}\right) & \leq \mathrm{P}\left(B_{i^{i}-S_{i}}\right)+\mathrm{P}\left(A_{i^{i}-2 S_{i}}\right) \mathrm{P}\left(C_{i^{i}-S_{i}}\right) \\
& \leq i\left(1-i^{-1}\right)^{S_{i}}+\mathrm{P}\left(A_{i^{i}-2 S_{i}}\right)\left(1-\left(i^{-1}\right)^{3 i / 8}\right) .
\end{aligned}
$$

Iterating $M_{i}$ times we obtain

$$
\mathrm{P}\left(A_{i^{i}}\right) \leq i\left(1-i^{-1}\right)^{S_{i}} M_{i}+\mathrm{P}\left(A_{i^{i}-M_{i} S_{i}}\right)\left(1-\left(i^{-1}\right)^{3 i / 8}\right)^{M_{i}},
$$

and, from this, (3.3) follows.

To complete the proof of Theorem 2.2 in the $\alpha=1$ case, we prove Lemma 3.3. 
Proof of Lemma 3.3. First we prove (3.4). Since $\alpha=1$, it holds that

$$
\frac{1}{i} \leq \min \left\{q_{1}^{N L}, q_{2}^{N L}, \ldots, q_{i^{i}}^{N L}\right\}
$$

and we have, for every $j<J_{i} \leq i^{i}$,

$$
\mathrm{P}(j \rightarrow j+1) \leq 1-q_{j}^{N L} \leq 1-\frac{1}{i} .
$$

Therefore,

$$
\mathrm{P}\left(j \rightarrow J_{i}\right)=\mathrm{P}(j \rightarrow j+1) \prod_{k=j+1}^{J_{i}-1} \mathrm{P}(j \rightarrow k+1 \mid j \rightarrow k) \leq\left(1-i^{-1}\right)^{J_{i}-j} .
$$

Hence, using countable subadditivity,

$$
\mathrm{P}\left(B_{J_{i}}\right) \leq \sum_{j=1}^{J_{i}-S_{i}-1}\left(1-i^{-1}\right)^{J_{i}-j} \leq\left(1-i^{-1}\right)^{S_{i}} \sum_{j=1}^{\infty}\left(1-i^{-1}\right)^{j} \leq i\left(1-i^{-1}\right)^{S_{i}} .
$$

This proves (3.4).

Next we prove (3.5). Observe that

$$
\begin{aligned}
\mathrm{P}\left(C_{J_{i}}\right) & =1-\prod_{j=J_{i}-S_{i}}^{J_{i}-1}\left[1-\mathrm{P}\left(j \rightarrow J_{i}\right)\right] \\
& \leq 1-\prod_{j=J_{i}-S_{i}}^{J_{i}-1}\left[1-\left(1-i^{-1}\right)^{J_{i}-j}\right] \\
& =1-\prod_{j=1}^{S_{i}}\left[1-\left(1-i^{-1}\right)^{j}\right] \\
& \leq 1-\prod_{j=1}^{\infty}\left[1-\left(1-i^{-1}\right)^{j}\right] .
\end{aligned}
$$

Thus, to prove (3.5), we have only to show that

$$
\prod_{j=1}^{\infty}\left[1-\left(1-i^{-1}\right)^{j}\right] \geq i^{-3 i / 8} .
$$

This formula is proved in three steps.

First we show that

$$
\prod_{j=1}^{i / 4}\left[1-\left(1-i^{-1}\right)^{j}\right] \geq i^{-i / 4}
$$

Using the fact that $\left(1-i^{-1}\right)^{j} \leq 1-i^{-1}$ for $j \geq 1$, we obtain

$$
\prod_{j=1}^{i / 4}\left[1-\left(1-i^{-1}\right)^{j}\right] \geq \prod_{j=1}^{i / 4} i^{-1}=i^{-i / 4}
$$

whence (3.7) holds. 
In the second step, to prove (3.6), we show that

$$
\prod_{j=i / 4+1}^{i-1}\left[1-\left(1-i^{-1}\right)^{j}\right]>\left(1-\mathrm{e}^{-1 / 4}\right)^{3 i / 4} \text {. }
$$

Using the fact that $\left(1-i^{-1}\right)^{i / 4+r} \leq\left(1-i^{-1}\right)^{i / 4} \leq \mathrm{e}^{-1 / 4}$ for $r \geq 0$, we have

$$
\prod_{i / 4+1}^{i-1}\left[1-\left(1-i^{-1}\right)^{j}\right] \geq \prod_{i / 4+1}^{i-1}\left(1-\mathrm{e}^{-1 / 4}\right) \geq\left(1-\mathrm{e}^{-1 / 4}\right)^{3 i / 4}
$$

This establishes (3.8).

In the third and final step, we use the fact that $-\mathrm{e} x \leq \ln (1-x)$ for $x \leq 1-\mathrm{e}^{-1}$ and $\left(1-i^{-1}\right)^{j} \leq \mathrm{e}^{-1} \leq 1-\mathrm{e}^{-1}$ for all $j \geq i$ to get

$$
\sum_{j=i}^{\infty} \ln \left(1-\left(1-i^{-1}\right)^{j}\right) \geq-\mathrm{e}\left(1-i^{-1}\right)^{i} \sum_{j=i}^{\infty}\left(1-i^{-1}\right)^{j-i} \geq-i .
$$

From this equation we conclude that

$$
\prod_{j=i}^{\infty}\left[1-\left(1-i^{-1}\right)^{j}\right] \geq \mathrm{e}^{-i}
$$

Finally, from (3.7), (3.8), and (3.9), for large enough $i$,

$$
\prod_{j=1}^{\infty}\left[1-\left(1-i^{-1}\right)^{j}\right]>i^{-i / 4}\left(1-\mathrm{e}^{-1 / 4}\right)^{3 i / 4} \mathrm{e}^{-i} \geq i^{-3 i / 8} .
$$

This establishes (3.6), completing the proof of (3.5) and consequently the proof of Lemma 3.3.

Theorem 2.2 is therefore proved for the case in which $\alpha=1$.

Now we sketch the proof in the case in which $\alpha>1$. Note the change in (3.3):

$$
\mathrm{P}\left(A_{i^{i}}\right) \leq i^{\alpha}\left(1-i^{-\alpha}\right)^{S_{i}} M_{i}+\left(1-\left(i^{-1}\right)^{3 i / 8}\right)^{M_{i}} .
$$

This equation is true in view of the next result.

Lemma 3.4. For large enough $i$,

$$
\begin{aligned}
& \mathrm{P}\left(B_{J_{i}}\right) \leq i^{\alpha}\left(1-i^{-\alpha}\right)^{S_{i}}, \\
& \mathrm{P}\left(C_{J_{i}}\right) \leq 1-\left(i^{-1}\right)^{3 i / 8} .
\end{aligned}
$$

Proof. The arguments are analogous to those used in Lemma 3.3. First, note that $\mathrm{P}(j \rightarrow$ $j+1) \leq 1-i^{-\alpha}$, and this implies that (3.11) holds. In addition,

$$
\begin{gathered}
\prod_{j=1}^{i(4 \alpha)^{-1}}\left[1-\left(1-i^{-\alpha}\right)^{j}\right] \geq i^{-i / 4}, \\
\prod_{j=i(4 \alpha)^{-1}+1}^{i^{\alpha}-1}\left[1-\left(1-i^{-\alpha}\right)^{j}\right]>\left(1-\mathrm{e}^{-1 / 4 \alpha^{2}}\right)^{i^{\alpha}-i(4 \alpha)^{-1}}, \\
\text { and } \prod_{j=i^{\alpha}}^{\infty}\left[1-\left(1-i^{-\alpha}\right)^{j}\right] \geq \mathrm{e}^{-i^{\alpha}}
\end{gathered}
$$

yield (3.12). 
Lemma 3.4 implies that (3.10) holds, in the same way that Lemma 3.3 is used to prove Lemma 3.2. Using (3.10), we conclude in the same manner that the independent particle process dies out a.s. in the $\alpha>1$ case. This establishes Theorem 2.2.

\section{Final remarks}

The next remark shows a possible generalization of Theorem 2.2. Its proof easily follows from the original proof.

Remark 4.1. The particle process on $\mathbb{Z}$ dies out a.s. if, for some $\alpha>0$ and some $f(i)$ such that $\lim _{i \rightarrow \infty} f(i)=\infty$,

$$
\frac{1}{(f(i))^{\alpha}} \leq \min \left\{q_{1}^{N L}, q_{2}^{N L}, \ldots, q_{f(i)}^{N L} f(i) .\right.
$$

From Remark 4.1 and Theorem 2.1, we get the following result.

Remark 4.2. Suppose that, for some $\alpha>0$ and some $f(i)$ such that

$$
\lim _{i \rightarrow \infty} f(i)=\infty
$$

we have

$$
\frac{1}{(f(i))^{\alpha}} \leq \min \left\{q_{1}^{N L}, q_{2}^{N L}, \ldots, q_{f(i)}^{N L}{ }^{f(i)}\right\}
$$

for large enough $i$. Then the sequence $\left(q_{i}\right)_{i \in \mathbb{N}}$ is such that $\sum_{i=1}^{\infty}\left(q_{i}\right)^{M}$ diverges for all $M>0$.

\section{Acknowledgements}

The authors would like to thank the anonymous referee for many helpful suggestions and remarks that improved the paper. EL and FPM are grateful to CNPq (311909/2009-4 and 306927/2007-1) and MZM thanks FACEPE (0126-1-02/06).

\section{References}

[1] Bertacchi, D. And Zucca, F. (2008). Critical behaviors and critical values of branching random walks on multigraphs. J. Appl. Prob. 45, 481-497.

[2] Bremaud, P. (1999). Markov chains. Gibbs fields, Monte Carlo Simulation, and Queues (Texts Appl. Math. 31). Springer, New York.

[3] Gantert, N. and Schmidt, P. (2009). Recurrence for the frog model with drift on $\mathbb{Z}$. Markov Process. Relat. Fields 15, 51-58.

[4] Kurtz, T. G., Lebensztayn, E., Leichsenring, A. R. and Machado, F. P. (2008). Limit theorems for an epidemic model on the complete graph. ALEA 4, 45-55.

[5] Lebensztayn, E., Machado, F. P. and Martinez, M. Z. (2008). Random walks systems with killing on $\mathbb{Z}$. Stochastics 80, 451-457. 